Errata

\title{
Erratum: Induced tensor in allowed nuclear beta decay
}

[Phys. Rev. C 4, 740 (1971)]

Barry R. Holstein

The second line of Eq. (11) should read

$$
\overrightarrow{\mathrm{A}}=-g_{A}\left(q^{2}\right) \sum_{i} \tau_{i}^{+} \vec{\sigma}_{i} e^{-\vec{i} \vec{q} \cdot \vec{r}},
$$

and the first line of Eq. (12) should read

$$
\begin{aligned}
A_{0}= & g_{A}(0) \frac{i \sum_{i} \tau_{i}^{+} \overline{\mathrm{q}} \cdot \vec{\sigma}_{i} \times \overrightarrow{\mathrm{L}}_{i}}{m_{p}+m_{n}} \\
& -\frac{1}{2} g_{A}(0) \sum_{i} \tau_{i}^{+}\left[H, \vec{\sigma}_{i} \cdot \overrightarrow{\mathrm{F}}_{i} \overrightarrow{\mathrm{q}} \cdot \overrightarrow{\mathrm{F}}_{i}\right] .
\end{aligned}
$$

\section{Erratum: Induced-tensor and second-order-forbidden terms}

[Phys. Rev. C 5, 1947 (1972)]

Barry R. Holstein

In Eq. (6) the factor $E_{0}+\frac{3}{2} E$ and in Eq. (8) the factor $E_{0}+\frac{5}{2} E$, both multiplying $\operatorname{Re} c^{*} j_{3}$, should be replaced by $\frac{7}{2} E$. In the expression for $\alpha_{+}$the final line should read

$$
\left.-\frac{6}{(35)^{1 / 2}} \operatorname{Re} c^{*} j_{3} \frac{E}{2 M}\right) \text {. }
$$

